\title{
Fracture Analysis of Propellant Grain under an Internal Pressure Based on Extended Finite Element Method
}

\author{
Li Zhang ${ }^{\text {a }}$, Jin-Zhao Huang and Zhi Lv \\ Department of Astronautic Science and Mechanics, Harbin Institute of Technology, China \\ a19666894@hit.edu.cn
}

Keywords: Extended Finite Element Method (XFEM). Crack propagation process. Internal pressure. Propellant grain

Abstract: The extended finite element method (XFEM) is a newly developed method for studying the fracture problems. With the XFEM, the crack is independent of the grids. Compared with the traditional finite element method, it avoids dividing the complex mesh near the crack tip and remeshing work when simulating crack propagation. Thus the calculation efficiency of the XFEM is high. In this paper, a solid rocket motor grain with initial crack under an internal pressure was studied by the XFEM. The characteristics of crack propagation was analyzed and some meaningful conclusions were drawn.

\section{Introduction}

When the solid rocket motor works, the internal temperature and pressure of the engine are rapidly increased due to the combustion of the propellant grain. The internal pressure of the engine usually reaches its peak in 0.1 seconds, and the internal pressure will gradually decrease in the following process (The internal pressure situation is shown in Fig. 1). Because of the thermal conductivity in the propellant grain is not high, the internal pressure in this stage is the dominant factor causing the damage of propellant grain. If the engine can withstand the impact of the maximum internal pressure and keep the integrity of the structure, in other stages, the failure probability will be very small. Therefore, the research on the crack propagation behavior of this process is of great significance to the design of the project. The reserach work using the XFEM to simulate the cracking process is still very limited. In this paper, a solid rocket motor grain with initial crack under an internal pressure was studied by the XFEM.

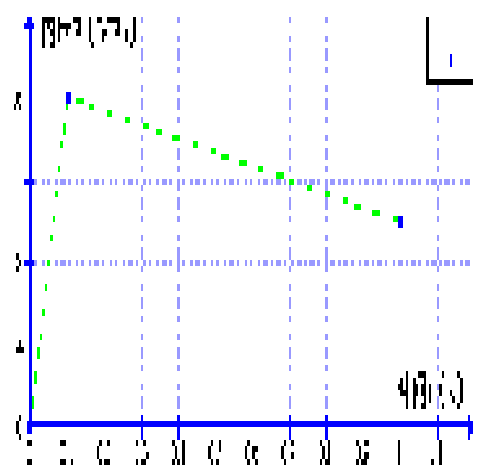

Fig.1 Condition of internal pressure

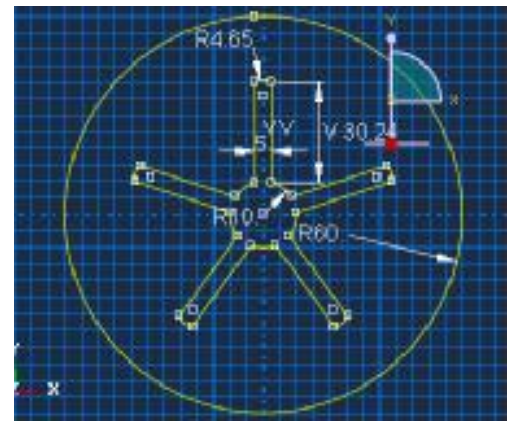

Fig.2 The size of grain

\section{Finite Element Model}

A large number of facts indicate that the positions of excessive arc are the most possible to emerge cracks in the propellant grain ${ }^{[1,2]}$. When calculating the crack, initial crack is usually added in this position. This paper selects the general pentagram propellant grain model. The radius of grain is $60 \mathrm{~mm}$. The radius of the pentagram in the root position is $10.05 \mathrm{~mm}$. The radius of excessive arc is $5 \mathrm{~mm}$. In order to increase the calculation speed, the length of the grain is only $40 \mathrm{~mm}$, the size is shown in Fig. 2. 
The initial crack is located in the position of a excessive arc. The calculation process is divided into 2 steps for high efficency. In the first step, we just need to build simple grids and the approximate range of crack propagation can be determined as shown in Fig.3. Fig.4 shows the calculation results when the crack is $1 \mathrm{~mm}$. It aims to illustrate the uncertainty in the direction of crack propagation. This also shows the need for the first step. In the second step, some grids are deleted and a special processing is conducted in this range to form the grids suitable for XFEM. 13343 8-node hexahedron elements are used. The grid division is detailed in Fig.5 to Fig.6.

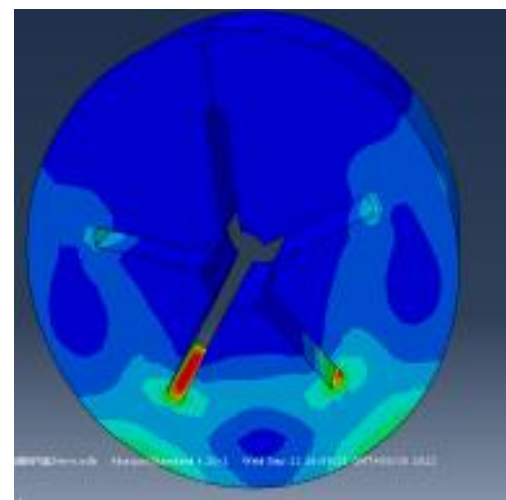

Fig.3 Determining the range of crack propagation

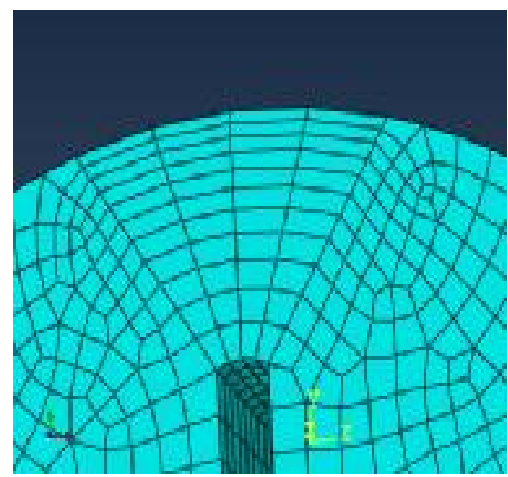

Fig.5 Meshs in crack area

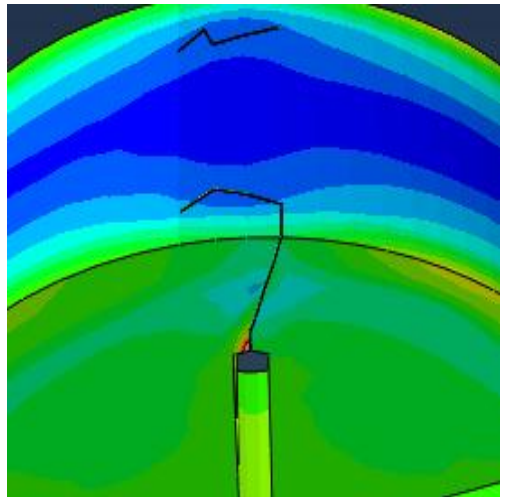

Fig.4 Crack propagation of the initial crack length

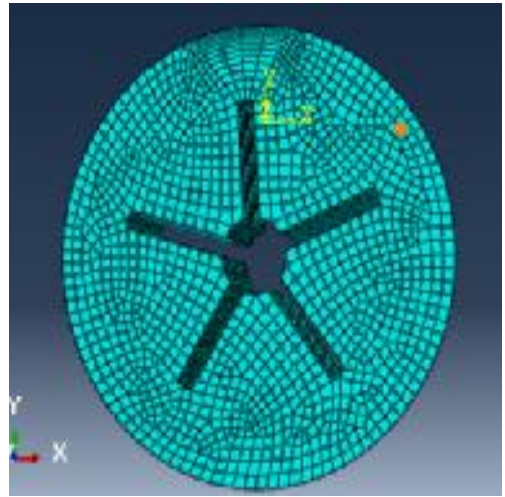

Fig.6 The whole meshs

\section{Crack propagation results}

In this section, we mainly study the parameter variation of the crack propagation and the crack propagation process.

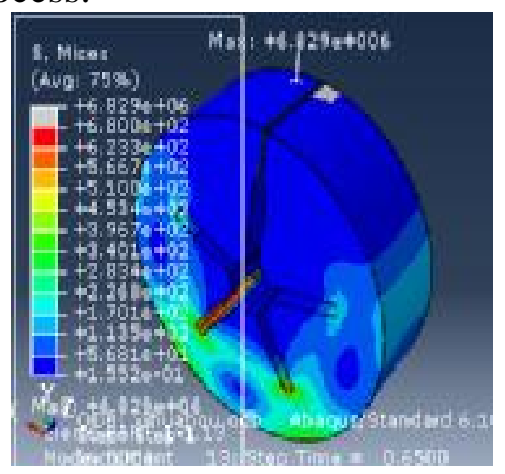

Fig.7 The whole stress distribution

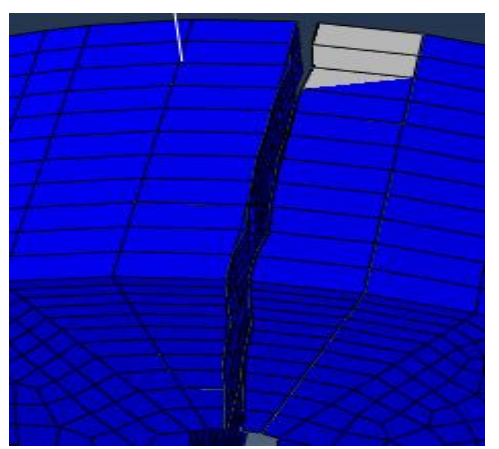

Fig. 8 The crack throughout the meshs

Under internal pressure load, the propellant grain containing initial crack damages very quickly. The crack extends to the edge of the grain in $0.65 \mathrm{~s}$. From the fracture surface we can see that the 
direction of the whole crack propagation process has not changed in general. From the stress distribution, the stress at the bottom of the grain is much larger than that of the middle part of the grain. The stress distribution of the two excessive arcs is compared by the method of displaying analysis results along the path. The path 1 is in the middle excessive arc path, the path 2 is in the bottom excessive arc.

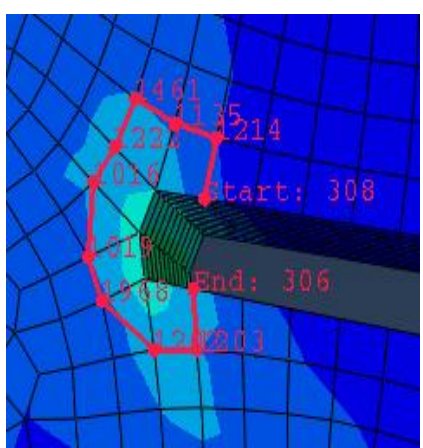

Fig.9 Path one

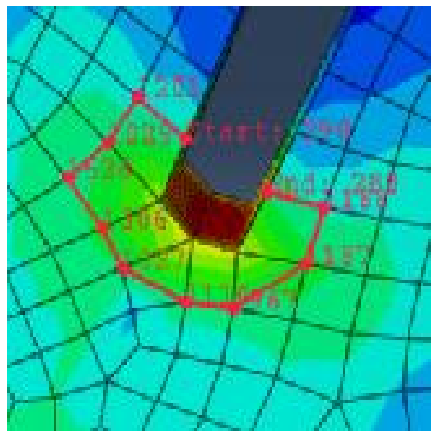

Fig.11 Path two

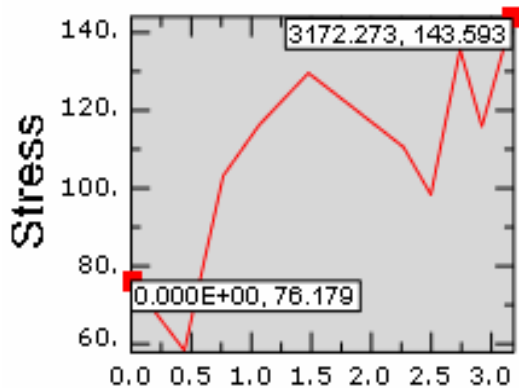

Fig.10 Stress change curve along path 1

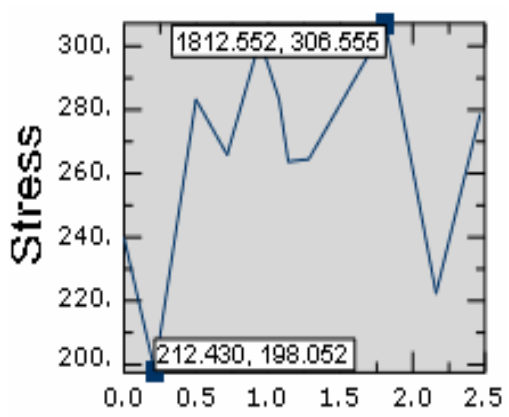

Fig.12 Stress change curve along path 2

As can be seen from the stress change curve, the minimum stress along the path 1 occurs at node 1214 and the maximum stress occurs at node 308 . The minimum stress along the path 2 occurs at node 1201 and the maximum stress occurs at node 1025. Comparing the two curve, it's not difficult to see that the stress at the bottom excessive arc significantly larger than the middle excessive arc. And the stress value in the vicinity of the crack is even smaller. Therefore, the farther away from the crack area, the less affected by the crack is and the greater the average stress distribution in the region is.

\section{Crack propagation process}

The steps of crack propagation are given as follows:

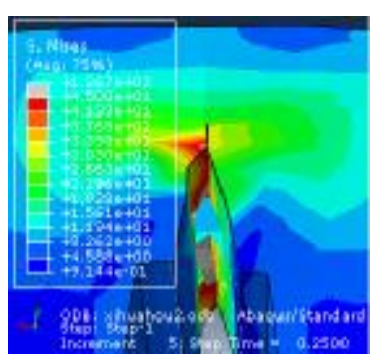

(1)

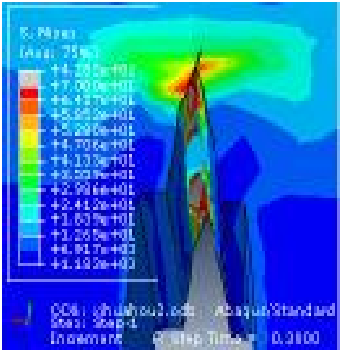

(2)
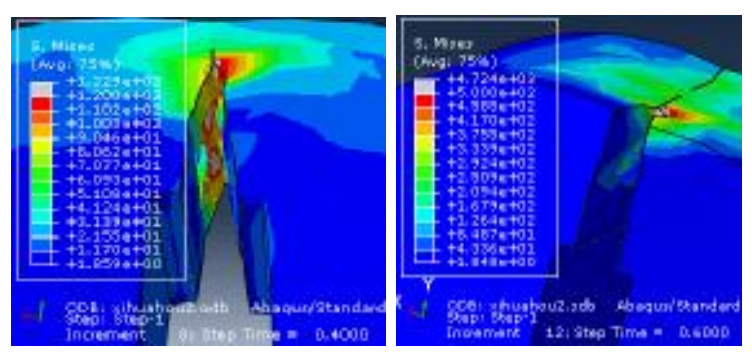

(3)
(4)

Fig.13 Crack propagation process 
Along with the crack propagation, the stress distribution near the crack tip is changed. Fig.13 (1) shows that the maximum stress around the crack is at the left side of the crack tip.In Fig.13(2), the maximum stress area is on both sides of the crack. From Fig.13(3) and (4), the maximum area of the crack is on the right side of the crack. And the phenomenon of concentration is becoming more and more obvious. This is mainly due to the change in the direction of crack propagation in each expansion step. It can found that with crack propagation, crack face becomes irregular and rugged. From Fig.13(4) it can be seen that there is a propagation trend towards the middle part when the crack propagas to the edge of the grain. The crack in two directions propagas at the same time until the grain damage.

In this paper, mainly the stress and some energy parameters of the crack tip are discussed. We select the nodes at the outer surface of the crack tip (or its nearest node). With each propagation step value, we get the image of stress at the crack tip.

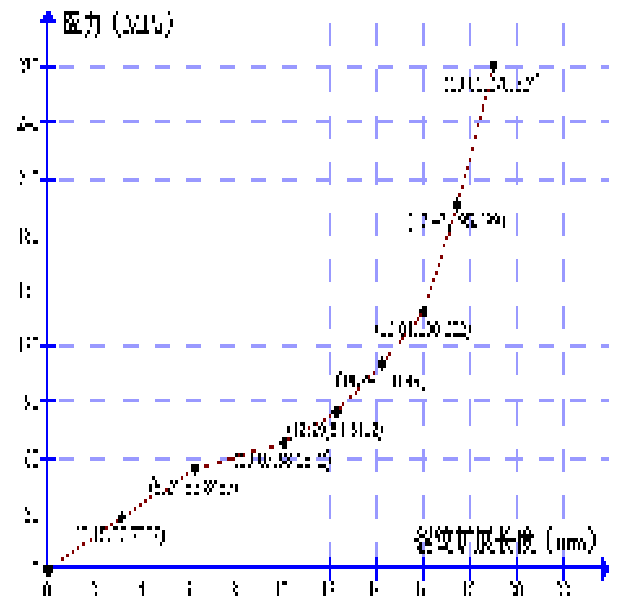

Fig.14 Crack tip stress varies with the crack propagation

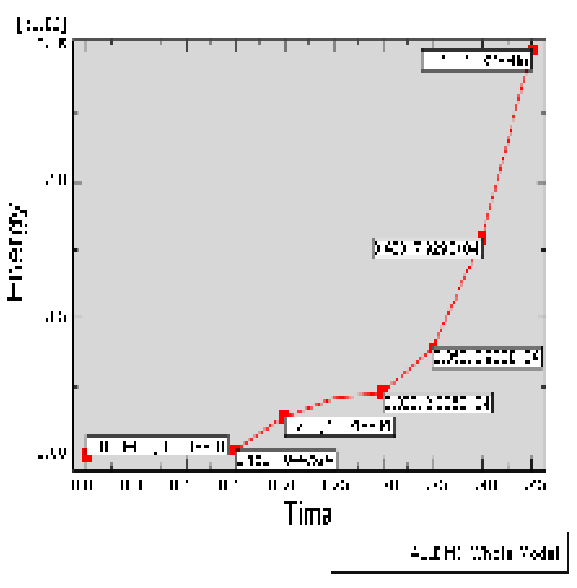

Fig.15 Damage dissipation energy

It can be seen from Fig.14 that the stress value of the crack tip increases with the crack propagation and increases faster and faster. This shows that the crack propagation ability is more and more strong. In postprocessing, we can get the curve of the damage dissipation energy by 0 s to $0.45 \mathrm{~s}$ in the whole model from the historical variable.

The value of the energy released during $0 s$ to $0.15 s$ is very small because this stage is the initial stage of the crack and also the first step of crack propagation. The energy released is $1.414 \times 10^{4} \mathrm{~N} \bullet \mathrm{mm}$ which is an order of magnitude higher than the first step. It shows that the energy release rate is obviously accelerated, and the propagation ability is obviously enhanced. The energy release speed is getting larger and larger with time. It shows that the energy release rate increases with time. It also shows that the crack propagation rate increases. The damage dissipation energy reachs maximum value in $0.45 \mathrm{~s}$ which is $1.482 \times 10^{5} \mathrm{~N} \bullet \mathrm{mm}$ and equal to $148.2 \mathrm{~J}$.

\section{Conclusion}

1) The crack has little effect on the stress of excessive arc at the far distance from the crack. From the whole grain stress distribution we can obtain that the influence degree of the crack stress depends on the distance between the node and the crack.

2) There is a phenomenon that the crack face is not smooth.

3) With the propagation of the crack, the rising rate of the crack tip stress value and crack propagation ability becomes large. And the failure rate is very fast, which shows the existence of the initial crack is very easy to cause the destruction of the rocket engine. 


\section{References}

[1] Bing Su, Zhong-Nian Shao. Analysis of stress and strain of solid propellant under the condition of ocean temperature [J]. Journal of Sichuan Ordnance, 2012, 04: 110-112.

[2] Shi-Jun Zhi. Crack analysis of solid rocket motor with viscoelastic grain. Master Thesis. Nanjing University of Science and Technology, 2009,05. 